\title{
Concha Roldán, Daniel Brauer, Johannes Rohbeck (eds.), Philosophy of Globalization, Berlín - Boston, Walter de Gruyter, 2018, 464 pp.
}

E ste libro reúne una serie de trabajos que representan los resultados de dos proyectos de investigación dirigidos por Concha Roldán (IFS-CSIC): "Philosophy of History and Globalisation of Knowledge. Cultural Bridges between Europe and Latin America" (WORLBRIDGES: F7-PEOPLE-2013-IRSES: PIRSESGA-2013-612644) y "The Philosophical-Moral Prisms of Crises. Towards a New Socio-Political Pedagogy" (PRISMAS: FFI2013-42935-). El eje de los autores es indagar la polivalencia semántica del término "globalización" y sus usos en la actualidad. Se divide en cinco capítulos.

Los trabajos reunidos en el primer capítulo analizan los cambios económicos y políticos que se producen en torno al proceso de la globalización, así como el efecto que esto tiene en la conducta de los individuos. Griselda Gutiérrez examina la nueva estructuración de los campos jurídicos, económicos, políticos, científicos y culturales bajo dicho proceso. Alejandro Alba aborda el mismo tema desde la evolución en este marco de la noción de ciudadano. Muestra que el aspecto legal-político, que lo definía en el pasado, resulta en la actualidad insuficiente y que es ahora necesario tener en cuenta el rol del individuo dentro de un mercado transnacional. A partir de esto, una nueva práctica puede sur- gir en el campo de la ciudadanía global, como la que desarrolla María Navarro, al referirse a la cognición cooperativa, práctica que promueve nuevas bases epistemológicas para recolectar información y analizar la realidad política desde nuevas perspectivas que no solo dependan del ámbito local-político. Todas estas dimensiones de la globalización no pueden ser comprendidas si dejamos de lado el hecho que presenta Franz Heilgendorff, esto es, la expansión del capitalismo occidental en la época de la globalización. En base a lo anterior, Constanze Demuth muestra cómo las naciones instituidas bajo el capitalismo utilizan estas nuevas formas de economía internacional para imponer sus agendas. Esta imposición aparece también en el ámbito bélico a través de las diferentes "esferas culturales", por lo que nos encontramos en una nueva instancia de choque de civilizaciones que ahora, como menciona Markus Tieddeman, se encuentran mediadas por dos universalismos, una versión suave y una crítica. Omar Acha arroja luz a esta última definición, señalando que esta es la única que busca reconocer las diferencias culturales sin intentar utilizarlas para la guerra o la conquista.

La segunda parte del libro trata sobre la justicia global dentro del ámbito de la globalización. Concha Roldán trata este tópico y propone la búsqueda 
de nuevos estándares éticos y políticos, mientras que Elisabetta Di Castro intenta extender esta visión de justicia global por medio de la instauración de nuevas instituciones que luchen por esta causa. Julia Muñoz amplía esta perspectiva proponiendo la posibilidad de un sistema de salud universal, abogando por este derecho desde una perspectiva contemporánea, que responda a las dificultades teóricas sobre el Estado, la caridad y la imposibilidad de ambos para abarcar un posible sistema sanitario global. Por otro lado, Alberto Ruiz comenta la problemática de la atribución de quienes son o deberían ser estos sujetos de derecho, pensando no solo en el presente sino en las consecuencias que la injusticia global va a traer en un futuro cercano, siendo esta noción expandida por Irene Franco hacia el campo de una justicia intergeneracional. Johannes Rohbeck cierra el capítulo a través de la reflexión acerca de la responsabilidad de los individuos y los estados con respecto al pasado y el efecto de esto en el presente y el futuro.

El capítulo tres nos remite a la historia de la globalización en distintas partes del mundo, mostrando los diferentes efectos durante los primeros estadios de este proceso, que tiene como punto de partida el descubrimiento de América. Abordando esta perspectiva, Nicholas Miller realiza un estudio sobre la influencia del colonialismo durante el siglo XIX. Por otro lado, Rolando Carrasco lleva a cabo un estudio sobre las misiones jesuitas como instauradoras de una identidad opuesta a la estereotípica sobre las tribus del Chaco, mostrando una faceta de una alteridad como algo positivo. A continuación, Sergio $\mathrm{Pa}-$ checo evalúa las consecuencias de las políticas ambientales y sanitarias que las élites de la ciudad de México realizaron durante el siglo XIX. En el texto de Darina Martykánová y Meltem Kocaman podemos ver cómo las condiciones históricas de Europa durante los siglos XVIII a XX dieron impulso a la migración de ingenieros de Europa occidental hacia tierras otomanas y como ello produjo un enriquecimiento $\mathrm{mu}-$ tuo. A la par de los avances en el campo de caminos, puentes y ciudades, el texto de Kaan Üçsu demuestra el poder de la globalización como herramienta para acelerar los avances tecnológicos y científicos de distintas ramas, en este caso, la cartografía en el ámbito del desarrollo bélico. Luego, Ridvan Turhan comenta el desarrollo intelectual y constitución de Turquía dentro del periodo de modernización del país luego de la Primera Guerra Mundial, buscando la independencia del imperialismo mundial a través de un proceso de industrialización. En el texto final de esta tercera parte, Agnieszka Pufelska realiza un análisis de la transición del feudalismo al capitalismo en Polonia a través de la visión de Witold Kula.

La parte cuatro inscribe la globalización en el marco de la historia de la filosofía, comenzando con el texto de Ricardo Aguilar, quien afirma que a partir de las nuevas formas de comunicación que surgen en esta era es la dicotomía deber-derecho la que hay que volver a estudiar bajo el contexto de la globalización para intentar responder si existe un sujeto de obligación moral hoy en día. Roberto Aramayo, por otra parte, analiza las críticas de Diderot hacia el colonialismo y la influencia que tuvo esta visión en la filosofia de Kant. También Efraín Lazos se ocupa de este último. Sostiene que fue uno de los primeros en teorizar los conceptos de hos- 
pitalidad y paz mundial, orientando estas nociones hacia un ámbito legal-político, y muestra que el concepto de globalización surge en el siglo XVIII, donde por primera vez se forja la idea de una sociedad cosmopolita. Luego, Iwan-Michelangelo D'Aprile intenta articular el concepto de globalización con la filosofia del siglo XVIII. Con ese fin, afirma que el concepto de ilustración debe ser ampliado no solo con respecto a su alcance temporal, sino también geográfico. Facundo Martín indaga desde una perspectiva marxista el concepto de "riqueza", en pos de hallar un aspecto emancipatorio bajo el contexto de las nuevas formas de desarrollo económico del capitalismo. Luego, Marco Kleber analiza la globalización a partir de la crítica de Heidegger a la Modernidad, analizando el mundo como una construcción metafisica del hombre basada en la relación sujeto-objeto. Desde un ángulo diferente, Lucas von Ramin estudia los fenómenos de la globalización en términos de formas simbólicas, buscando alertar y dar cuenta de la forma contingente de estos fenómenos y su dependencia de cierta forma de comprensión del mundo, cultura y tiempo en el que nos hallamos.

La última sección del libro investiga cómo la filosofía de la historia puede contribuir para un análisis sistemático del fenómeno de la globalización. Daniel Brauer propone la noción de "Historia Global", que abarca diferentes cambios en el campo historiográfico, buscando explicar, interpretar y dar cuenta de la interdependencia de diferentes eventos históricos que traspasan la barrera nación-estado para colocarse en una dimensión global. Intenta, de esta manera, restablecer herramientas tradicionales de la filosofia de la historia para estudiar estos cambios en la sociedad y en las categorías históricas contemporáneas. Luego, Elías Palti indaga la teoría sobre la historia de Koselleck, a partir de la crítica que realiza Foucault acerca de las multiplicidades de formas que la noción de temporalidad y sujeto fueron tomando, tanto antes como después del Sattelzeit (1750-1850), dando lugar a una reconfiguración de las nociones de temporalidad de la modernidad y el presente. A continuación, Adrián Ratto propone evaluar el proceso de globalización a la luz de la filosofía de la Ilustración, dejando de lado ciertos estereotipos que se han forjado acerca de esta. Desde otra perspectiva filosófica, Francisco Naishtat indaga las críticas de Heidegger y Benjamin a la Modernidad. Por último, Rosa Belvedresi realiza una investigación en torno a la noción de "futuro" como categoría de tiempo histórico y el revés práctico de esta noción como guía y nexo entre el presente y el porvenir.

Para concluir, el texto reúne una serie de trabajos que, con rigor intelectual y desde diferentes enfoques, examinan el proceso de "globalización", sus raíces y sus consecuencias. El trabajo resulta una lectura recomendable no solo para politólogos, sociólogos y filósofos de la historia especializados en el tema, sino también para todos aquellos interesados en un examen analítico de los problemas del presente y el futuro.

MARCELO ESCALANTE $U B A$ 